\title{
AMIGO2 as a novel indicator of liver metastasis in patients with colorectal cancer
}

\author{
AKIMITSU TANIO $^{1}$, HIROAKI SAITO $^{1,2}$, MASATAKA AMISAKI $^{1}$, KAZUSHI HARA $^{1}$, KEN SUGEZAWA $^{1}$, \\ CHIHIRO UEJIMA ${ }^{1}$, YOICHIRO TADA ${ }^{1}$, KYOICHI KIHARA ${ }^{1}$, MANABU YAMAMOTO ${ }^{1}$, KANAE NOSAKA ${ }^{3}$, \\ RYO SASAKI $^{4}$, MITSUHIKO OSAKI ${ }^{4,5}$, FUTOSHI OKADA ${ }^{4,5}$ and YOSHIYUKI FUJIWARA ${ }^{1}$ \\ ${ }^{1}$ Division of Gastrointestinal and Pediatric Surgery, Department of Surgery, School of Medicine, Faculty of Medicine, \\ Tottori University, Yonago, Tottori 683-8504; ${ }^{2}$ Department of Surgery, Japanese Red Cross Tottori Hospital, Yonago, \\ Tottori 680-8517; ${ }^{3}$ Division of Organ Pathology, Faculty of Medicine; ${ }^{4}$ Division of Experimental Pathology, \\ Faculty of Medicine; ${ }^{5}$ Chromosome Engineering Research Center, Tottori University, Yonago, Tottori 683-8503, Japan
}

Received March 11, 2020; Accepted November 10, 2020

DOI: 10.3892/ol.2021.12539

\begin{abstract}
Our previous study showed that adhesion molecule with immunoglobulin like domain 2 (AMIGO2) is a pivotal driver gene of liver metastasis via regulating tumor cell adhesion to liver endothelial cells in mouse models. The aim of the present study was to clarify the role of AMIGO2 in liver metastasis in patients the colorectal cancer (CRC). Two human CRC cell lines, Caco-2 (AMIGO2-low) and HCT116 (AMIGO2-high), were used in this study. AMIGO2-overexpressing Caco-2 and AMIGO2-knockdown HCT116 cells were generated by transfection with an AMIGO2 expression vector or AMIGO2 small interfering RNA, respectively. Cell proliferation, invasion and adhesion to human liver endothelial cells were examined in in vitro studies. Immunohistochemical analysis was also performed to evaluate the association between AMIGO2 expression and liver metastasis in patients with CRC. In vitro studies revealed that cell proliferation, invasion and adhesion to liver endothelial cells were accelerated by upregulation of AMIGO2 expression, but suppressed by downregulation of $\mathrm{AMIGO} 2$ expression in human $\mathrm{CRC}$ cells. Immunohistochemical analysis using clinical CRC specimens revealed that $\mathrm{AMIGO} 2$ expression was associated with the frequency of liver metastasis $(\mathrm{P}<0.01)$, but not that of pulmonary metastasis $(\mathrm{P}=0.611)$ and peritoneal dissemination
\end{abstract}

Correspondence to: Dr Hiroaki Saito, Department of Surgery, Japanese Red Cross Tottori Hospital, 117 Shotoku-cho, Yonago, Tottori 680-8517, Japan

E-mail: sai10@tottori-med.jrc.or.jp

Abbreviations: AMIGO2, adhesion molecule with Ig like domain 2; BET, bromodomain and extra terminal domain; CRC, colorectal cancer; HHSECs, human hepatic sinusoidal endothelial cells; PTK7, tyrosine-protein kinase-like 7; siRNA, small interfering RNA

Key words: AMIGO2, CRC, liver metastasis, recurrence
$(\mathrm{P}=0.909)$. In addition, AMIGO2 expression levels in tumor cells were significantly higher in liver metastatic foci than primary lesions $(\mathrm{P}=0.012)$. In conclusion, the present results indicated that AMIGO2 expression may contribute to the formation of liver metastasis in CRC.

\section{Introduction}

Colorectal cancer (CRC) is one of the most common malignancies worldwide. Although recent advances in surgical techniques, chemotherapy, and molecular targeted drugs have improved the prognosis of CRC patients, it ranks fourth among all cancer-related deaths worldwide (1). Liver metastasis is the most frequently observed metastasis site and the strongest determinant for prognosis in CRC patients. It occurs in 50\% of patients during follow-up for CRC after surgery (2) and is responsible for two-thirds of CRC patient deaths (3). Therefore, it is extremely important to develop new treatments for liver metastasis to improve the prognosis of CRC patients. To this end, it is indispensable to understand the detailed mechanism by which CRC cells metastasize to the liver.

The adhesion molecule with immunoglobulin like domain (AMIGO) family of molecules was identified as novel transmembrane proteins that are involved in neuronal processes by a homophilic binding mechanism (4). It was reported that $\mathrm{AMIGO} 2$ inhibits apoptosis and promotes the survival of cerebellar granule neurons (5). Recently, Kanda et al (6) revealed that $\mathrm{AMIGO} 2$ functions as a driver gene for liver metastasis in mouse models. In fact, they demonstrated that knockdown of AMIGO2 expression in highly liver metastatic mouse fibrosarcoma cells in vitro and in vivo resulted in the suppression of liver metastasis via attenuation of tumor cell adhesion to hepatic vascular endothelial cells. Conversely, forced expression of AMIGO2 in non-metastatic parental fibrosarcoma cells induced an increase in hepatic vascular endothelial cell adhesion and hepatic metastasis of tumor cells (6). Furthermore, Huo et al (7) recently reported that upregulated AMIGO2 expression became evident as CRC advanced, as detected by transcriptome analysis using The 
Cancer Genome Atlas. These results encouraged us to determine the role of AMIGO2 in terms of liver metastasis from $\mathrm{CRC}$. Therefore, the aim of this study was to clarify the role of AMIGO2 in liver metastasis in CRC.

\section{Materials and methods}

Cell lines. Caco-2 was purchased from DS Pharma Biomedical (Osaka, Japan) and HCT116 was purchased from the American Type Culture Collection. Caco-2 cells were maintained in EMEM (051-07615; Wako) supplemented with $10 \%$ fetal bovine serum (FBS; P30-3306; PAN-Biotech $\mathrm{GmbH}), 5 \%$ non-essential amino acids (139-15651; Wako), penicillin, and streptomycin (168-23191; Wako). HCT116 cells were maintained in McCoy's 5A (16600-082; Gibco; Thermo Fisher Scientific, Inc.) supplemented with 10\% FBS, penicillin, and streptomycin. Human hepatic sinusoidal endothelial cells (HHSECs) were purchased from ScienCell Research Laboratories, Inc. (cat. no. 5000) and maintained with Endothelial Cell Medium (cat. no. 1001; ScienCell Research Laboratories, Inc.) supplemented with 5\% FBS and Endothelial Cell Growth Supplement (cat. no. 1052; ScienCell Research Laboratories, Inc.). Cultures were maintained at $37^{\circ} \mathrm{C}$ in an atmosphere of $95 \%$ air and $5 \% \mathrm{CO}_{2}$.

Western blot analysis. Cells were lysed in protein extraction buffer (28941279; GE Healthcare) containing protease inhibitor (80650123; GE Healthcare) to obtain whole cell lysates. Protein concentrations were determined using a Bradford protein assay. Proteins were separated by $10 \%$ SDS-PAGE, and then transferred onto $0.2 \mu \mathrm{m}$ PVFD membranes (1704156; Bio-Rad). Following incubation in 5\% skimmed milk, the membranes were reacted with mouse monoclonal anti-AMIGO2 antibody (1:500, clone G-7, sc-373699; Santa Cruz Biotechnology, Inc.) or with mouse monoclonal anti- $\beta$-actin antibody $(1: 5,000$, clone AC-15, A5441; Sigma-Aldrich; Merck KGaA), and then with peroxidase-conjugated sheep monoclonal anti-mouse $\mathrm{IgG}$ antibody (1:3,000, NA931; GE Healthcare) in 5\% skimmed milk.

Transfection. The pEZ-M02-AMIGO2 expression vector (EX-Mm13004-M02) was purchased from GeneCopoeia. An empty vector used as a control was generated by removing the Amigo 2 insert by restriction digest as follows. The pEZ-M02-AMIGO2 plasmid was digested with EcoRI and NotI (R0101S and R0189L, respectively; New England Biolabs). Then 5 'overhangs were filled using KOD DNA polymerase (KOD-101; Toyobo) to generate blunt ends, which were ligated together using Ligation high (LGK-101; Toyobo). Caco-2 cells were transfected with the AMIGO2 expression vector (OE-AMIGO2) or the empty vector (OE-Empty) using Lipofectamine ${ }^{\circledR} 2000$ (12566014; Invitrogen; Thermo Fisher Scientific, Inc.) and two stable clones for OE-AMIGO2 (OE-AMIGO2-Caco-2-1 and OE-AMIGO2-Caco-2-2) and OE-Empty (OE-Empty-Caco-2-1 and OE-Empty-Caco-2-2) were selected in the presence of $750 \mu \mathrm{g} / \mathrm{ml} \mathrm{G}-418$ sulfate (074-05963; Wako). HCT116 cells were transfected with $0.67 \mu \mathrm{M}$ siRNA targeting AMIGO2 (4392420; Ambion) or with negative control siRNA (4390843; Ambion) using Lipofectamine RNAiMAX (13778100; Invitrogen; Thermo Fisher Scientific, Inc.).
Proliferation assay. The proliferation of CRC cells was evaluated using the CCK-8 assay. Briefly, CRC cells in $200 \mu \mathrm{l}$ culture medium were seeded in three wells of a 96-well plate at a density of 5,000 cells/well. After $72 \mathrm{~h}, 10 \mu \mathrm{l}$ CCK-8 assay solution (347-07621; Dojindo Molecular Technologies, Inc.) was added to each well and the cells were incubated for $60 \mathrm{~min}$ at $37^{\circ} \mathrm{C}$ in an atmosphere of $95 \%$ air and $5 \% \mathrm{CO}_{2}$. The absorbance was determined at $450 \mathrm{~nm}$ against a reference wavelength of $620 \mathrm{~nm}$ using a microplate reader (Infinite F50R; Tecan). The mean value of three wells was used for statistical analysis.

Invasion assay. Cell invasion assays were performed using BioCoat Matrigel invasion chambers (BD Biosciences) in accordance with the manufacturer's protocol. Briefly, CRC cells $\left(1 \times 10^{5}\right)$ were seeded in the inserts of Matrigel-coated invasion chambers ( 24 wells, $8-\mu \mathrm{m}$ pore size) filled with serum-free DMEM medium. Then, the cells were incubated with DMEM medium containing 20\% FBS in the lower chamber at $37^{\circ} \mathrm{C}$ in an atmosphere of $95 \%$ air and $5 \% \mathrm{CO}_{2}$. After $24 \mathrm{~h}$, non-migrating cells were removed from the top of the filter with a cotton swab. The invading cells at the bottom of the filter were fixed with methanol for $10 \mathrm{~min}$ and stained with $0.2 \%$ crystal violet and then counted using a microscope (ECLIPSE Ts2; Nikon) in three different visual fields (magnification, $\mathrm{x} 100$ ). The mean value of three different fields was used for statistical analysis.

Adhesion assay. Tumor cell adhesion assays were performed in accordance with previous reports $(6,8)$. Briefly, a 96-well plate (165305; Thermo Fisher Scientific, Inc.) was coated with $1 \%$ gelatin (071-06291; Wako) for $16 \mathrm{~h}$. A total of $8 \times 10^{3}$ HHSECs were seeded in each well after removal of the gelatin solution. Tumor cells $\left(2 \times 10^{5}\right)$ labeled with the PKH67 green fluorescent dye (PKH67GL-1KT; Sigma-Aldrich; Merck KGaA) were then placed onto HHSEC monolayers in the wells and incubated for $30 \mathrm{~min}$. The non-adherent cells were removed by washing with PBS, and the adherent cells were quantified with a fluorescent plate reader (Infinite M200 PRO; Tecan) at an excitation of $485 \mathrm{~nm}$ and an emission of $535 \mathrm{~nm}$. The percentage of adherence was calculated as the fluorescence ratio (post-wash fluorescence/pre-wash fluorescence x100).

Patient samples. Immunohistochemical analysis was performed using paraffin-embedded CRC samples from 267 patients with CRC who underwent proctocolectomies at our institution between January 2007 and December 2015. Normal colorectal tissues were available in 119 patients out of 267 patients in which immunohistochemical analysis was performed. Clinicopathological findings were determined by the Japanese Classification of Colorectal Carcinoma (9). None of the patients had received radiotherapy, chemotherapy, or other medical interventions before surgery.

Immunohistochemistry. Tissue samples were fixed in formalin and embedded in paraffin. Serial sections were cut at $4 \mu \mathrm{m}$, deparaffinized in xylene, and rehydrated through a graded alcohol series. For retrieval of AMIGO2, the sections were boiled for $20 \mathrm{~min}$ in a microwave oven in $10 \mathrm{mM}$ citrate buffer ( $\mathrm{pH}$ 6.0). The samples were incubated in $3 \%$ hydrogen 


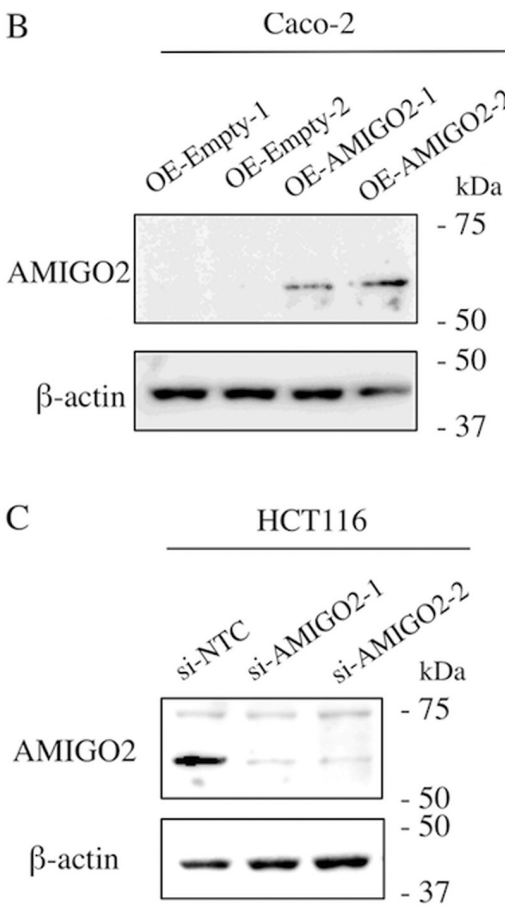

Figure 1. Western blot analysis of AMIGO2. (A) AMIGO2 expression in Caco-2 and HCT116 cells. (B) Transfection of EX-Mm13004-M02 increased AMIGO2 expression in Caco-2 cells. (C) Transfection of siRNA targeting AMIGO2 decreased AMIGO2 expression in HCT-116 cells. AMIGO2, adhesion molecule with Ig like domain 2; siRNA, small interfering RNA; OE, overexpression; NTC, non-targeting control.

peroxidase for 30 min to block endogenous peroxidases and in Block Ace (UK-B80; DS Pharma Biomedical) for $30 \mathrm{~min}$ to prevent non-specific antigen binding. The slides were subsequently incubated with primary antibodies (mouse anti-AMIGO2, 1:400; Santa Cruz Biotechnology, Inc.) overnight at $4^{\circ} \mathrm{C}$ and then incubated with Envision+ Dual Link (K4063; Dako) for $30 \mathrm{~min}$. Staining was visualized with diaminobenzidine (SK-4105; Vector Laboratories) and the sections were counterstained with hematoxylin. The expression of AMIGO2 in CRC cells was evaluated in a blinded manner. In brief, five fields were chosen at random and examined at $\mathrm{x} 400$ magnification. The staining intensity on the cell surfaces of CRC cells was scored as 0 (negative), 1 (weak), or 2 (moderate to strong) as previously reported (6). This evaluation was based on the comparison of AMIGO2 expression of lymphocytes in the tissue of CRC since AMIGO2 is expressed in T-cells (10).

Collection of data of AMIGO2 mRNA expression in CRC patients. The data of AMIGO2 mRNA expression in CRC patients were obtained from The Cancer Genome Atlas (TCGA) Research Network (http://cancergenome.nih.gov/) through The Human Protein Atlas (https://www.proteinatlas. org) on April 20, 2020. Five hundred and seventy seven patients, in whom both survival data and stage of disease are available, were used for survival analysis.

Statistical analysis. The data of proliferation, invasion, and adhesion were checked for normality with Shapiro Wilk test. Differences in proliferation, invasion, and adhesion were evaluated using the Kruskal-Wallis test and Dunn's test. Differences between categorical variables were determined using the $\chi^{2}$ test. Differences in AMIGO2 expression between normal tissues and cancer tissue and between the tissues obtained from liver metastasis of CRC and their primary lesions were determined with the Wilcoxon test. Univariate and multivariate analyses to identify the risk factors of liver metastasis were performed by logistic regression analysis and a stepwise procedure. Survival curves were calculated using the Kaplan-Meier method and differences between survival curves were examined using the log-rank test. $\mathrm{P}<0.05$ was considered significant. GraphPad Prism 6 (GraphPad Software, Inc.) and SPSS statistics version 24.0 (IBM Corp.) software were used for the statistical analyses.

\section{Results}

AMIGO2 expression in CRC cell lines. We first assessed the expression of AMIGO2 in CRC cell lines by western blotting and found that it was low in Caco-2 and high in HCT116 cells (Fig. 1A). We then determined the efficacy of transfection of EX-Mm13004-M02 and siRNA targeting AMIGO2 in CRC cells. EX-Mm13004-M02 was able to increase AMIGO2 expression in Caco-2 cells (Fig. 1B). Furthermore, siRNA targeting AMIGO2 could efficiently decrease AMIGO2 expression in HCT116 cells (Fig. 1C).

AMIGO2 regulates $C R C$ cell proliferation and invasion. We next determined the effect of AMIGO2 on the proliferation of CRC cell lines by CCK-8 assay. The proliferation of OE-AMIGO2-Caco-2 cells was significantly higher than that of OE-Empty-Caco-2 cells ( $\mathrm{P}=0.002$, Fig. 2A). The proliferation of si-AMIGO2-HCT116 cells was significantly less than that of si-NTC-HCT116 cells ( $\mathrm{P}=0.007$, Fig. 2B). Regarding the invasion of CRC cells, the invasive ability of OE-AMIGO2-Caco-2 cells was significantly more than that of OE-Empty-Caco-2 
A

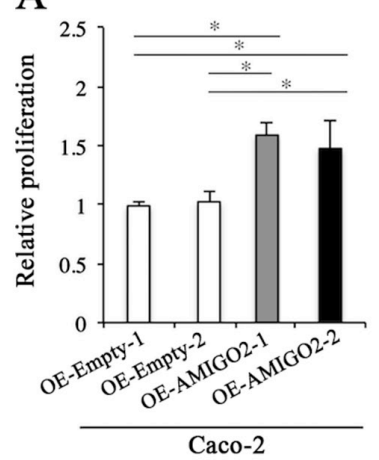

E

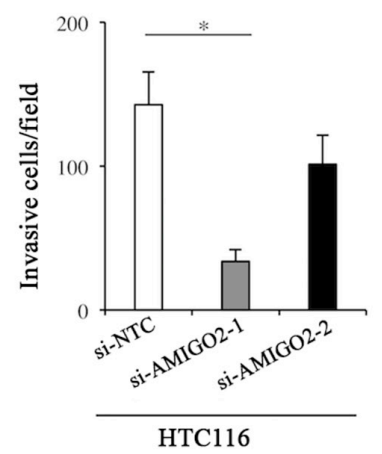

B

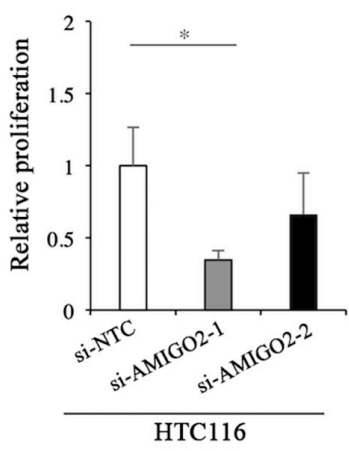

F

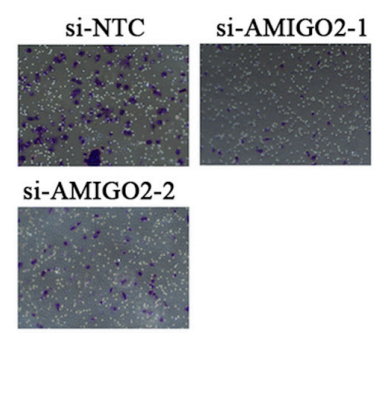

C

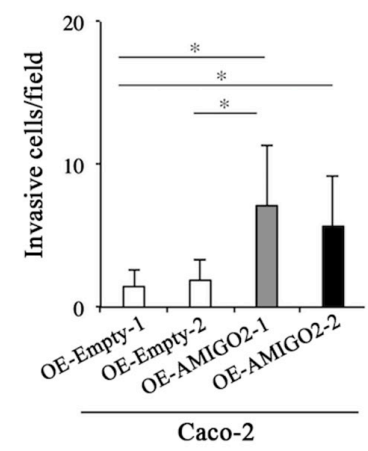

G

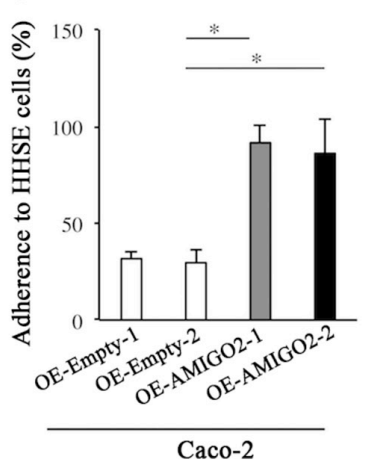

D

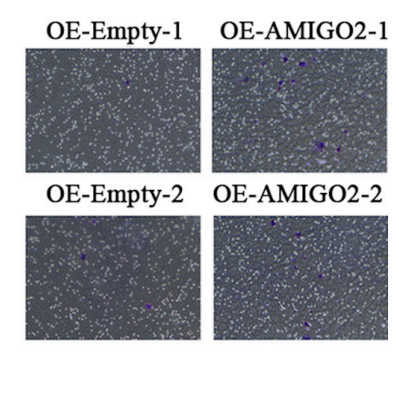

$\mathrm{H}$

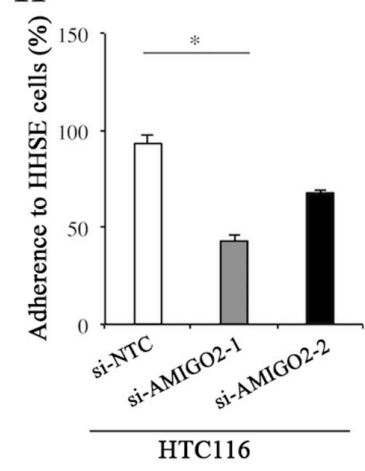

Figure 2. Proliferation, invasion, and adhesion assays. (A) The proliferation of Caco-2 cells transfected with EX-Mm13004-M02 was significantly higher than that of Caco-2 cells transfected with empty vector. (B) The proliferation of HCT-116 cells transfected with siRNA targeting AMIGO2 was significantly lower than that of HCT-116 transfected with negative control siRNA. (C) The invasive ability of Caco-2 cells transfected with EX-Mm13004-M02 was significantly greater than that of Caco-2 cells transfected with empty vector. (D) The representative images for the invasion assay in each condition of (C) Magnification, $\mathrm{x} 100$. (E) The invasive ability of HCT116 cells transfected with siRNA targeting AMIGO2 was significantly less than that of HCT116 cells transfected with negative control siRNA. (F) The representative images for the invasion assay in each condition of (E) Magnification, x100. (G) Caco-2 cells transfected with EX-Mm13004-M02 demonstrated significantly increased adhesion to human hepatic sinusoidal endothelial cells (HHSECs), when compared with controls. (H) HCT116 cells transfected with AMIGO2 siRNA demonstrated significantly reduced adhesion to HHSECs, when compared with controls. The data were checked for normality with Shapiro Wilk test. Differences in proliferation, invasion, and adhesion were evaluated using the Kruskal-Wallis test and Dunn's test. "significant difference between 2 groups by Dunn's test $(\mathrm{P}<0.05)$. AMIGO2, adhesion molecule with Ig like domain 2; siRNA, small interfering RNA; OE, overexpression; NTC, non-targeting control; HHSE, human hepatic sinusoidal endothelial.

cells $(\mathrm{P}<0.001$, Fig. 2C and D). Furthermore, the invasive ability of si-AMIGO2-HCT116 cells was significantly less than that of si-NTC-HCT116 cells $(\mathrm{P}<0.001$, Fig. 2E and F). These results indicated that $\mathrm{AMIGO} 2$ regulated the proliferation and invasive ability of CRC cells.

AMIGO2 mediates the adhesion of CRC cells to human hepatic sinusoidal endothelial cells. We then determined the propensity for CRC cells to adhere to HHSECs. OE-AMIGO2-Caco-2 cells demonstrated significantly increased adhesion to HHSECs, when compared with OE-Empty-Caco-2 cells ( $\mathrm{P}=0.001$, Fig. 2G). Furthermore, si-AMIGO2-HCT116 cells demonstrated significantly decreased adhesion to HHSECs, when compared with si-NTC-HCT116 cells ( $\mathrm{P}<0.001$, Fig. $2 \mathrm{H})$. These results indicate that $\mathrm{AMIGO} 2$ regulates the adhesion of CRC cells to HHSECs.

AMIGO2 expression in CRC tissue. We then determined AMIGO2 expression in CRC tissue (Fig. 3). The staining intensity of AMIGO2 on the surfaces of CRC cells was scored as 0 (negative), 1 (weak), or 2 (moderate to strong) as previously reported (6) (Fig. 3A-C). The intensity of AMIGO2 staining was significantly stronger in cancer tissue, when compared with normal tissue ( $\mathrm{P}<0.001$, Fig. 3D).
Table I shows the association between AMIGO2 expression in CRC tissue and clinicopathological characteristics. AMIGO2 expression was observed more frequently in differentiated tumor than in undifferentiated tumor $(\mathrm{P}=0.001)$.

Regarding the association between AMIGO2 expression in CRC tissue and the site of metastasis, including both synchronous and metachronous metastasis, AMIGO2 expression in CRC tissue was significantly related to liver metastasis $(\mathrm{P}<0.001$, Fig. 4A), but not to pulmonary metastasis $(\mathrm{P}=0.61$, Fig. 4B) and peritoneal metastasis ( $\mathrm{P}=0.91$, Fig. 4C). Univariate analysis indicated that sex, lymph node metastasis, vascular invasion, and AMIGO2 expression in CRC tissue were factors associated with liver metastasis. Multivariate analysis also revealed that $\mathrm{AMIGO} 2$ expression in $\mathrm{CRC}$ tissue was an independent predictor of liver metastasis, along with sex, lymph node metastasis, and vascular invasion (Table II). Furthermore, we determined the association between AMIGO2 expression in CRC tissue and metachronous liver metastasis and found that $\mathrm{AMIGO} 2$ expression in CRC tissue was also significantly related to metachronous liver metastasis ( $\mathrm{P}<0.001$, Fig. 4D). Univariate analysis indicated that lymph node metastasis and AMIGO2 expression in CRC tissue were factors associated with metachronous liver metastasis (Table III). Multivariate analysis also revealed that $\mathrm{AMIGO} 2$ expression in $\mathrm{CRC}$ tissue 
Table I. Association between AMIGO2 expression and clinicopathological features.

\begin{tabular}{|c|c|c|c|c|}
\hline \multirow[b]{2}{*}{ Variables } & \multicolumn{3}{|c|}{ AMIGO2 expression } & \multirow[b]{2}{*}{ P-value } \\
\hline & $0(\mathrm{n}=82)(\%)$ & $1(\mathrm{n}=126)(\%)$ & $2(\mathrm{n}=59)(\%)$ & \\
\hline \multicolumn{5}{|l|}{ Age, years } \\
\hline$<70(\mathrm{n}=127)$ & $35(27.6)$ & $68(53.5)$ & $24(18.9)$ & 0.137 \\
\hline$\geq 70(n=140)$ & $47(33.6)$ & $58(41.4)$ & $35(25.0)$ & \\
\hline \multicolumn{5}{|l|}{ Sex } \\
\hline Male $(n=146)$ & $48(32.9)$ & $64(43.8)$ & $34(23.3)$ & 0.480 \\
\hline Female $(n=121)$ & $34(28.1)$ & $62(51.2)$ & $25(20.7)$ & \\
\hline \multicolumn{5}{|l|}{ Tumor location } \\
\hline Colon $(n=190)$ & $62(32.6)$ & $84(44.2)$ & $44(23.2)$ & 0.306 \\
\hline Rectum (n=77) & $20(26.0)$ & $42(54.5)$ & $15(19.5)$ & \\
\hline \multicolumn{5}{|l|}{ Tumor size, $\mathrm{cm}$} \\
\hline$<4.0(\mathrm{n}=103)$ & $34(33.0)$ & $45(43.7)$ & $24(23.3)$ & 0.659 \\
\hline$\geq 4.0(\mathrm{n}=164)$ & $48(29.3)$ & $81(49.4)$ & $35(21.3)$ & \\
\hline \multicolumn{5}{|l|}{ Histology $\mathrm{y}^{\mathrm{a}}$} \\
\hline Differentiated $(n=235)$ & $63(26.8)$ & $115(48.9)$ & $57(42.3)$ & 0.001 \\
\hline Undifferentiated $(\mathrm{n}=32)$ & $19(59.4)$ & $11(34.4)$ & $2(6.2)$ & \\
\hline \multicolumn{5}{|l|}{ Depth of invasion ${ }^{\mathrm{b}}$} \\
\hline $\mathrm{T} 1 / \mathrm{T} 2(\mathrm{n}=19)$ & $5(26.3)$ & $9(47.4)$ & $5(26.3)$ & 0.863 \\
\hline T3/T4 (n=248) & $77(31.0)$ & $117(47.2)$ & $54(21.8)$ & \\
\hline \multicolumn{5}{|l|}{ Lymph node metastasis } \\
\hline Absent $(n=139)$ & $46(33.1)$ & $67(48.2)$ & $26(18.7)$ & 0.348 \\
\hline Present $(n=128)$ & $36(28.1)$ & $59(46.1)$ & $33(25.8)$ & \\
\hline \multicolumn{5}{|l|}{ Lymphatic invasion $^{c}$} \\
\hline ly0/1 (n=105) & $32(30.5)$ & $52(49.5)$ & $21(20.0)$ & 0.761 \\
\hline ly $2 / 3(n=162)$ & $50(30.9)$ & $74(45.7)$ & $38(23.4)$ & \\
\hline \multicolumn{5}{|l|}{ Vascular invasion $^{\mathrm{d}}$} \\
\hline $\mathrm{v} 0 / 1 \quad(\mathrm{n}=159)$ & $45(28.3)$ & $81(50.9)$ & $33(20.8)$ & 0.327 \\
\hline $\mathrm{v} 2 / 3(\mathrm{n}=108)$ & $37(34.2)$ & $45(41.7)$ & $26(24.1)$ & \\
\hline
\end{tabular}

${ }^{a}$ Histology: Differentiated, well or moderately differentiated adenocarcinoma; Undifferentiated, poorly differentiated adenocarcinoma, signet ring cell carcinoma or mucinous carcinoma. ${ }^{\mathrm{b}} \mathrm{T} 1$, tumor invasion of the lamina propria or submucosa; $\mathrm{T} 2$, tumor invasion of the muscularis propria; T3, tumor invasion of the sub serosa or within adventitia; T4, tumor penetration of the serosa or tumor invasion of adjacent organs. 'Lymphatic invasion: ly0-ly3, grade of lymphatic invasion. ${ }^{\mathrm{d}}$ Vascular invasion: v0-v3, grade of vascular invasion. $\chi^{2}$ test was used for the statistical analysis. AMIGO2, adhesion molecule with Ig like domain 2.

was an independent predictor of metachronous liver metastasis, along with lymph node metastasis (Table III). Furthermore, AMIGO2 expression in CRC tissue was also significantly related to metachronous liver metastasis in node-negative patients $(\mathrm{P}<0.001$, Fig. $4 \mathrm{E})$, but not node-positive patients $(\mathrm{P}=0.085$, Fig. 4F).

Finally, we determined AMIGO2 expression in the tissues obtained from liver metastasis of CRC. We compared the intensity of AMIGO2 between a primary CRC lesion (Fig. 5A) and a matched liver metastatic lesion (Fig. 5B). The intensity of AMIGO2 staining was significantly stronger in the tissue obtained from the liver metastasis of CRC, when compared with the primary lesion $(n=21, P=0.012$, Fig. $5 C)$. With regard to the association between AMIGO2 expression in CRC tissue and prognosis of CRC patients, the prognosis of patients with high AMIGO2 mRNA expression in CRC tissue was significantly worse than that of patients with low AMIGO2 mRNA expression in $\mathrm{CRC}$ tissue ( $\mathrm{P}=0.013$, Fig. 5D).

\section{Discussion}

Metastasis is a multi-stage process that is collectively termed the invasion-metastasis cascade, in which cancer cells: i) Proliferate and locally invade through the surrounding extracellular matrix and stromal cell layers; ii) intravasate into the lumina of blood vessels; iii) survive the rigors of transport through the vasculature; iv) arrest at distant organ sites; v) extravasate into the parenchyma of distant tissues; vi) initially survive in these foreign microenvironments to form micrometastases; and vii) re-initiate their proliferative 
A

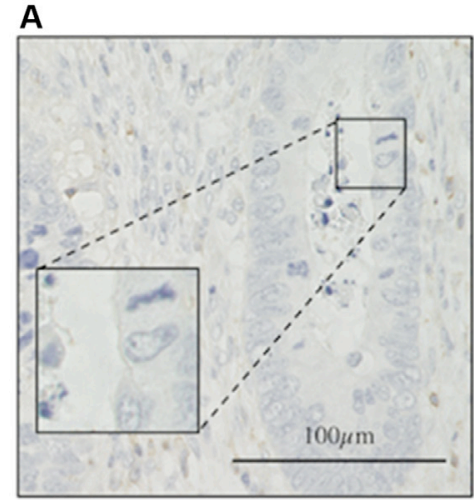

C

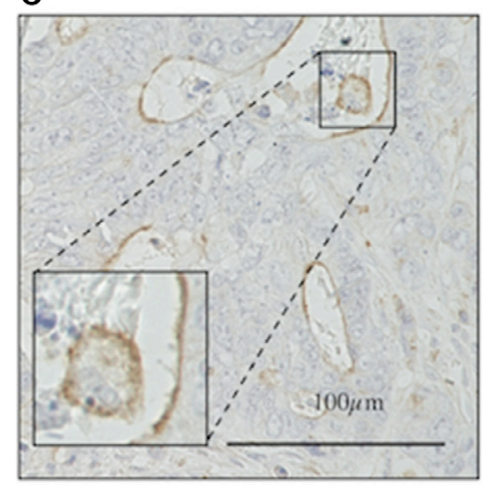

B

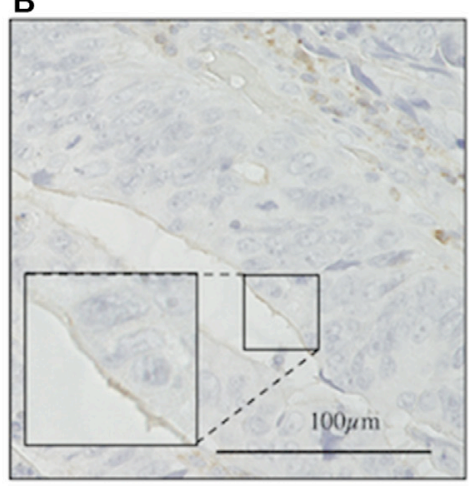

D

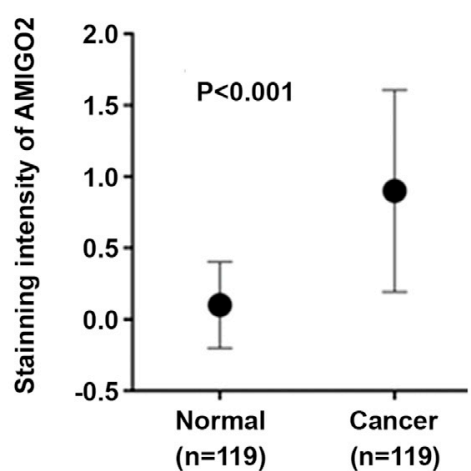

Figure 3. Immunohistochemistry. Representative pictures of AMIGO2 cell surface staining in colorectal cancer cells for the different intensity scores used in this study. (A) Score 0 (negative), (B) score 1 (weak), and (C) score 2 (moderate to strong). (D) The intensity of AMIGO2 staining was significantly stronger in cancer tissue, when compared with normal tissue $(\mathrm{P}<0.001)$. The Wilcoxon test was used for the statistical analysis. AMIGO2, adhesion molecule with Ig like domain 2.
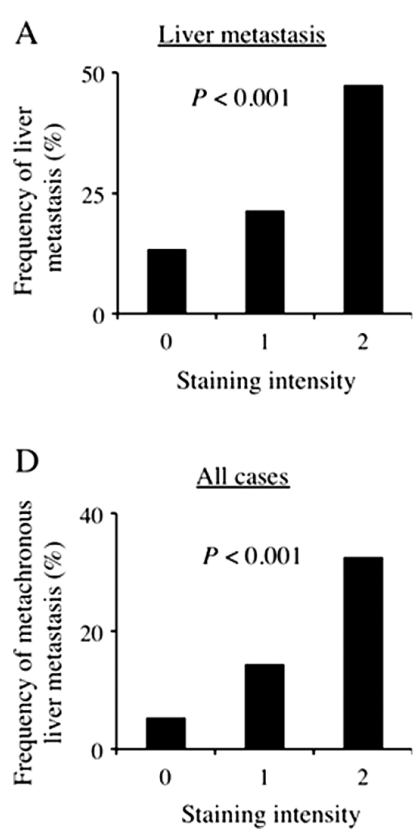

\section{$\mathrm{B}$}

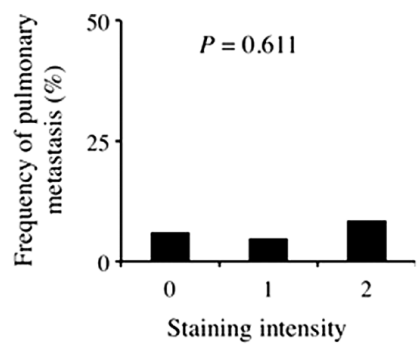

$\mathrm{E}$

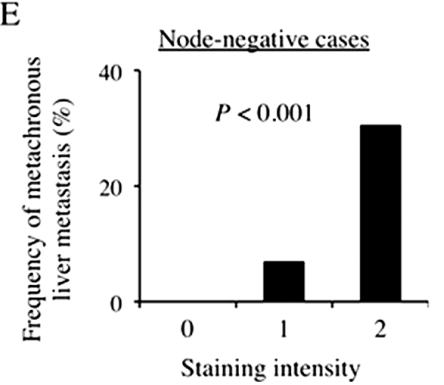

$\mathrm{C}$

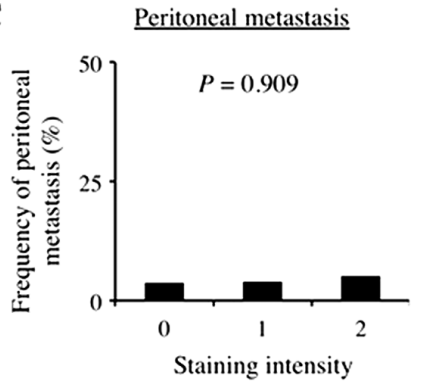

$\mathrm{F}$

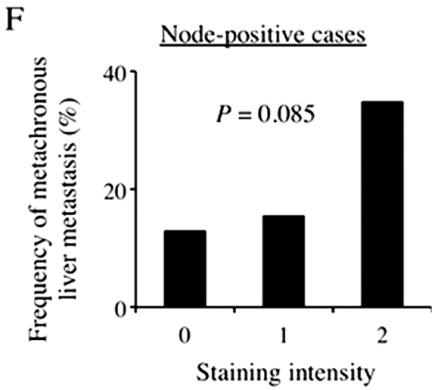

Figure 4. AMIGO2 expression on colorectal cancer cells and sites of metastasis. Increased AMIGO2 expression was significantly associated with increased frequency of (A) liver metastasis, but not (B) pulmonary metastasis and (C) peritoneal metastasis. (D) Increased AMIGO2 expression was significantly associated with increased frequency of metachronous liver metastasis in all patients. Increased AMIGO2 expression was significantly associated with increased frequency of metachronous liver metastasis in (E) node-negative patients, but not in (F) node-positive patients. $\chi^{2}$ test was used for all statistical analysis. AMIGO2, adhesion molecule with Ig like domain 2.

programs at metastatic sites, thereby generating macroscopic, clinically detectable neoplastic growths. These processes are orchestrated by molecular pathways operating within carcinoma cells. Among these seven processes, we first demonstrated 
Table II. Univariate and multivariate analyses of risk factors for liver metastases in patients with colorectal cancer.

\begin{tabular}{|c|c|c|c|c|c|c|}
\hline \multirow[b]{2}{*}{ Variables } & \multicolumn{3}{|c|}{ Univariate analysis } & \multicolumn{3}{|c|}{ Multivariate analysis } \\
\hline & P-value & $\mathrm{HR}$ & $95 \% \mathrm{CI}$ & P-value & HR & $95 \% \mathrm{CI}$ \\
\hline Age ( $<70$ vs. $\geq 70$ years) & 0.459 & 1.234 & $0.707-2.153$ & & & \\
\hline Sex (female vs. male) & 0.025 & 1.941 & $1.085-3.473$ & 0.012 & 2.260 & $1.200-4.254$ \\
\hline Tumor location (colon vs. rectum) & 0.992 & 1.003 & $0.543-1.854$ & & & \\
\hline Tumor size (<4.0 vs. $\geq 4.0 \mathrm{~cm})$ & 0.113 & 1.618 & $0.892-2.934$ & & & \\
\hline Histology (differentiated vs. undifferentiated) & 0.210 & 0.528 & $0.195-1.433$ & & & \\
\hline Depth of invasion (pT1/2 vs. pT3/4) & 0.155 & 2.957 & $0.665-13.151$ & & & \\
\hline Lymph node metastasis (absent vs. present) & 0.004 & 2.340 & $1.318-4.155$ & 0.014 & 2.165 & $1.170-4.006$ \\
\hline Lymphatic invasion (ly0/1 vs. ly2/3) & 0.252 & 1.407 & $0.785-2.522$ & & & \\
\hline Vascular invasion (v0/1 vs. v2/3) & 0.008 & 2.150 & $1.223-3.778$ & 0.010 & 2.220 & $1.206-4.088$ \\
\hline AMIGO2 (0 vs. 1 vs. 2 ) & $<0.001$ & 2.511 & $1.651-3.817$ & $<0.001$ & 2.585 & $1.677-3.985$ \\
\hline
\end{tabular}

$\mathrm{HR}$, hazard ratio; CI, confidence interval; AMIGO2, adhesion molecule with Ig like domain 2. Logistic regression analysis was used for the statistical analysis.

Table III. Univariate and multivariate analyses of risk factors for metachronous liver metastases in patients with colorectal cancer.

\begin{tabular}{|c|c|c|c|c|c|c|}
\hline \multirow[b]{2}{*}{ Variables } & \multicolumn{3}{|c|}{ Univariate analysis } & \multicolumn{3}{|c|}{ Multivariate analysis } \\
\hline & P-value & HR & $95 \% \mathrm{CI}$ & P-value & HR & $95 \% \mathrm{CI}$ \\
\hline Age ( $<70$ vs. $\geq 70$ years) & 0.432 & 1.363 & $0.629-2.957$ & & & \\
\hline Sex (female vs. male) & 0.449 & 1.344 & $0.625-2.888$ & & & \\
\hline Tumor location (colon vs. rectum) & 0.984 & 1.009 & $0.438-2.321$ & & & \\
\hline Tumor size $(<4.0 \mathrm{vs.} \geq 4.0 \mathrm{~cm})$ & 0.341 & 1.477 & $0.661-3.300$ & & & \\
\hline Histology (differentiated vs. undifferentiated) & 0.564 & 0.690 & $0.196-2.429$ & & & \\
\hline Depth of invasion (pT1/2 vs. pT3/4) & 0.311 & 2.772 & $0.356-21.603$ & & & \\
\hline Lymph node metastasis (absent vs. present) & 0.027 & 2.431 & $1.107-5.341$ & 0.040 & 2.357 & $1.042-5.332$ \\
\hline Lymphatic invasion (ly0/1 vs. ly2/3) & 0.105 & 2.022 & $0.863-4.741$ & & & \\
\hline Vascular invasion (v0/1 vs. v2/3) & 0.318 & 1.475 & $0.687-3.167$ & & & \\
\hline AMIGO2 (0 vs. 1 vs. 2$)$ & $<0.001$ & 3.175 & $1.785-5.733$ & $<0.001$ & 3.151 & $1.729-5.742$ \\
\hline
\end{tabular}

HR, hazard ratio; CI, confidence interval; AMIGO2, adhesion molecule with Ig like domain 2. Logistic regression analysis was used for the statistical analysis.

that $\mathrm{AMIGO} 2$ was closely related to the proliferation and invasion of CRC cells in the current study. With regards to the function of AMIGO2, it was reported to be involved in cell survival and angiogenesis via AKT signaling (11). However, it remains unclear whether AMIGO2 is involved in cell survival and angiogenesis via AKT signaling in CRC. It has recently been reported that $\mathrm{AMIGO} 2$ is upregulated in malignant melanoma, and that $\mathrm{AMIGO} 2$ and its interactor tyrosine-protein kinase-like 7 (PTK7) regulate the proliferation and survival of tumor cells in this disease (12). PTK7 is also known as colon carcinoma kinase 4. This gene is thought to be expressed in CRC but not in normal colon, and therefore may be involved in CRC progression (13-15). Although there is no report regarding the correlation between AMIGO2 and PTK7 in CRC thus far, it is likely that AMIGO2 regulates the proliferation of CRC cells through PTK7. With regards to invasion, Sonzogni et al (16) recently reported AMIGO2 as a new mediator of invasion in breast cancer. In the current study, we demonstrated that AMIGO2 knockdown in HCT116 cells suppressed invasive behavior. Furthermore, its overexpression in Caco-2 cells was sufficient to induce invasion, demonstrating that AMIGO2 also has important roles in the invasiveness of CRC cells.

Our study also demonstrated that AMIGO2 was closely associated with the adhesion of CRC cells to HHSECs. Furthermore, AMIGO2 expression on cancer cells in CRC tissue was related to increased frequency of liver metastasis. Of importance is that AMIGO2 expression on cancer cells in CRC tissue was not related to lung and peritoneal metastasis. In this regard, Kanda et al (6) demonstrated that the adhesion of LV12 cells, an isolated subline of QRsP-11 fibrosarcoma cells with high liver-metastatic properties, to liver endothelial cells was 
A

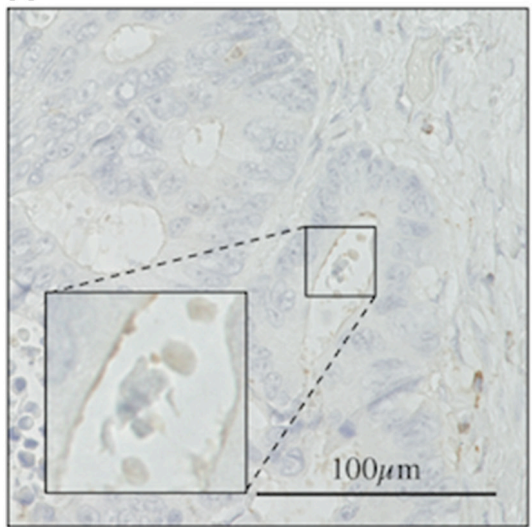

B

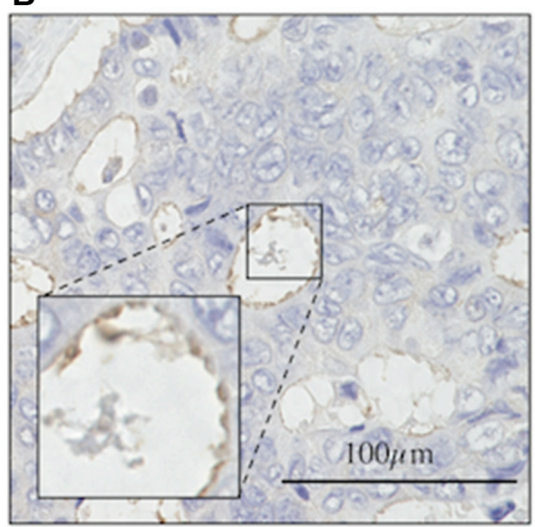

C

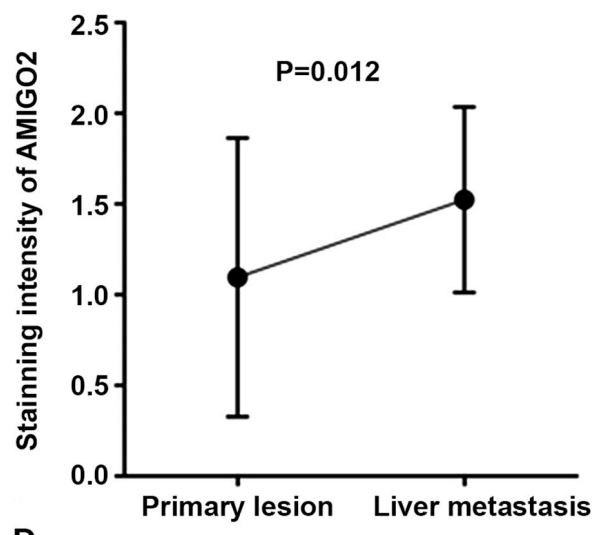

D

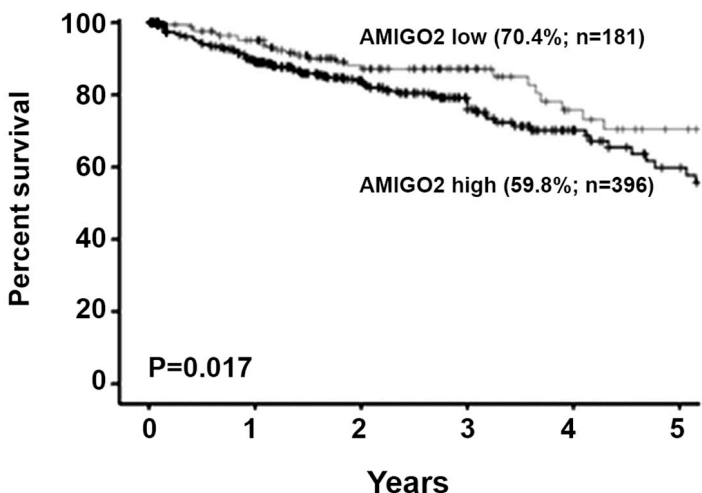

Figure 5. Representative images of AMIGO2 expression in (A) primary lesion (A) and (B) liver metastatic lesion obtained from the same patient. (C) The intensity of AMIGO2 staining was significantly stronger in the tissue obtained from liver metastasis of CRC compared with the primary lesion (n=21). The Wilcoxon test was used for the statistical analysis. (D) Kaplan-Meier analysis of overall survival in colon cancer patients with high ( $\geq 2.97$ FPKM) vs. low AMIGO2 (<2.97 FPKM) mRNA expression. The cut-off value was according to FPKM provided by The Human Protein Atlas. The differences between survival curves were examined using the log-rank test. FPKM, fragments per kilobase of exon per million reads mapped. AMIGO2, adhesion molecule with Ig like domain 2.

significantly higher than that of QRsP-11 cells, whereas adhesion to lung endothelial cells (LE-1) was similar in both cell lines. They also showed that the incidence of liver metastasis was higher following intravenous injection of LV12 cells than following injection of QRsP-11 cells. However, there was no difference in the incidence of lung metastasis between the two cell lines, suggesting that LV12 cells have a higher capacity to colonize the liver. Finally, they revealed that AMIGO2, which was overexpressed in LV12 cells, functioned as a driver gene for liver metastasis. We also found that AMIGO2 was more strongly expressed on the surfaces of cells in liver metastasis lesions compared with its expression in the primary lesions of human CRC in this study. These results may verify the involvement of AMIGO2 expression in the formation of liver metastases in clinical samples. However, the precise mechanisms by which selective and firm adhesion to liver endothelial cells, but not lung endothelial cells, can be brought about by AMIGO2 expression remain unclear. One possible mechanism to explain the AMIGO2-mediated cell adhesion is homophilic or heterophilic binding among the three homologous proteins that comprise the AMIGO family, i.e., AMIGO1, AMIGO2, and AMIGO3 (4). Regarding this, Kanda et al (6) showed that all these AMIGO family molecules are expressed on liver endothelial cells; however, none of these AMIGO molecules are expressed on LE-1 lung endothelial cells. Further experiments are required to resolve these mechanisms.
Of critical importance is the question of whether our results will prove useful to the diagnosis and treatment of CRC patients. With respect to the clinical usefulness of AMIGO2 in $\mathrm{CRC}$, this study revealed that $\mathrm{AMIGO} 2$ was an independent predictive factor of metachronous liver metastasis. The resection of liver metastasis improves the prognosis of CRC patients. A recent study demonstrated more than 60 months of median overall survival in CRC patients with up to four liver metastases who underwent surgery combined with perioperative FOLFOX4. It is likely that early detection of metachronous liver metastasis increases the resection rates of liver metastasis, improving the prognosis of CRC patients. Our results also demonstrated that lymph node metastasis was a useful predictive indicator of metachronous liver metastasis. Of importance is that AMIGO2 expression was a useful predictive indicator of metachronous liver metastasis even in node-negative CRC patients. Therefore, evaluation of AMIGO2 in resected specimens by immunohistochemistry might be useful in detecting node-negative CRC patients who have a high possibility of metachronous liver metastasis and need intensive follow-up and adjuvant chemotherapy after surgery, which may improve the prognosis of CRC patients.

Regarding treatment, a recent study demonstrated that AMIGO2 was upregulated in melanoma cells and tissues compared with human melanocytes and nevi, and AMIGO2 silencing in melanoma cells induces G1/S arrest followed by 
apoptosis. Furthermore, a BET inhibitor was shown to be able to silence AMIGO2 expression (12). Considering the multifunctional aspects of AMIGO2 shown in the current study, it is likely that BET inhibition may be useful in the treatment of $\mathrm{CRC}$ patients with high AMIGO2 expression. Considering the close association between AMIGO2 expression on CRC cells and liver metastasis observed in the current study, treatment directly targeting AMIGO2 might be effective in preventing liver metastasis in $\mathrm{CRC}$, eventually improving the prognosis of CRC patients.

In conclusion, AMIGO2 contributes to the formation of liver metastasis by regulating CRC cell adhesion to human hepatic sinusoidal endothelial cells, as well as the proliferation and invasion of CRC cells. Treatments that target AMIGO2 could therefore provide a novel form of CRC treatment.

\section{Acknowledgements}

The authors would like to thank Dr H. Nikki March for editing a draft of this manuscript.

\section{Funding}

This work was supported by grants from Takeda Pharmaceutical Company Limited.

\section{Availability of data and materials}

The datasets used and/or analyzed during the current study are available from the corresponding author upon reasonable request.

\section{Authors' contributions}

HS, AT, MA, KN, MO, FO and YF designed the study and wrote the paper. AT and RS carried out experiments. AT, KH, KS, CU, YT, KK and MY analyzed clinical data of patients with colorectal cancer. All authors have read and approved the final manuscript.

\section{Ethics approval and consent to participate}

The study protocol was approved by the Institutional Review Board at Tottori University Hospital (approval number: 1705A039). All procedures were in accordance with the ethical standards of the responsible committee on human experimentation (institutional and national) and with the Helsinki Declaration of 1964 and later versions. Written informed consent to be included in the study was obtained from all patients.

\section{Patient consent for publication}

Not applicable.

\section{Competing interests}

The authors declare that they have no competing interests.

\section{References}

1. Torre LA, Bray F, Siegel RL, Ferlay J, Lortet-Tieulent J and Jemal A: Global cancer statistics, 2012. CA Cancer J Clin 65: 87-108, 2015

2. Matsuoka H, Morise Z, Tanaka C, Hayashi T, Ikeda Y, Maeda K, Masumori K, Koide Y, Katsuno H, Tanahashi Y, et al: Repeat hepatectomy with systemic chemotherapy might improve survival of recurrent liver metastasis from colorectal cancer-a retrospective observational study. World J Surg Oncol 17: 33, 2019.

3. Abdalla EK, Adam R, Bilchik AJ, Jaeck D, Vauthey JN and Mahvi D: Improving resectability of hepatic colorectal metastases: Expert consensus statement. Ann Surg Oncol 13: 1271-1280, 2006

4. Kuja-Panula J, Kiiltomaki M, Yamashiro T, Rouhiainen A and Rauvala H: AMIGO, a transmembrane protein implicated in axon tract development, defines a novel protein family with leucine-rich repeats. J Cell Biol 160: 963-973, 2003.

5. Ono T, Sekino-Suzuki N, Kikkawa Y, Yonekawa H and Kawashima S: Alivin 1, a novel neuronal activity-dependent gene, inhibits apoptosis and promotes survival of cerebellar granule neurons. J Neurosci 23: 5887-5896, 2003.

6. Kanda Y, Osaki M, Onuma K, Sonoda A, Kobayashi M, Hamada J, Nicolson GL, Ochiya T and Okada F: Amigo2-upregulation in tumour cells facilitates their attachment to liver endothelial cells resulting in liver metastases. Sci Rep 7: 43567, 2017.

7. Huo T, Canepa R, Sura A, Modave F and Gong Y: Colorectal cancer stages transcriptome analysis. PLoS One 12: e0188697, 2017.

8. Onuma K, Suenaga Y, Sakaki R, Yoshitome S, Sato Y, Ogawara S, Suzuki S, Kuramitsu Y, Yokoyama H, Murakami A, et al: Development of a quantitative bioassay to assess preventive compounds against inflammation-based carcinogenesis. Nitric Oxide 25: 183-194, 2011.

9. Japanese Society for Cancer of the Colon and Rectum: Japanese classification of colorectal carcinoma, 2nd English edition. Kanehara \& Co., Ltd, 2009.

10. Li Z, Khan MM, Kuja-Panula J, Wang H, Chen Y, Guo D, Chen ZJ, Lahesmaa R, Rauvala H and Tian L: AMIGO2 modulates $\mathrm{T}$ cell functions and its deficiency in mice ameliorates experimental autoimmune encephalomyelitis. Brain Behav Immun 62: 110-123, 2017.

11. Park H, Lee S, Shrestha P, Kim J, Park JA, Ko Y, Ban YH, Park DY, Ha SJ, Koh GY, et al: AMIGO2, a novel membrane anchor of PDK1, controls cell survival and angiogenesis via Akt activation. J Cell Biol 211: 619-637, 2015.

12. Fontanals-Cirera B, Hasson D, Vardabasso C, Di Micco R, Agrawal P, Chowdhury A, Gantz M, de Pablos-Aragoneses A, Morgenstern A, Wu P, et al: Harnessing BET inhibitor sensitivity reveals AMIGO2 as a melanoma survival gene. Mol Cell 68: 731-744.e9, 2017.

13. Saha S, Sparks AB, Rago C, Akmaev V, Wang CJ, Vogelstein B, Kinzler KW and Velculescu VE: Using the transcriptome to annotate the genome. Nat Biotechnol 20: 508-512, 2002.

14. Tian X, Yan L, Zhang D, Guan X, Dong B, Zhao M and Hao C: PTK7 overexpression in colorectal tumors: Clinicopathological correlation and prognosis relevance. Oncol Rep 36: 1829-1836, 2016.

15. Lhoumeau AC, Martinez S, Boher JM, Monges G, Castellano R, Goubard A, Doremus M, Poizat F, Lelong B, de Chaisemartin C, et al: Overexpression of the promigratory and prometastatic PTK7 receptor is associated with an adverse clinical outcome in colorectal cancer. PLoS One 10: e0123768, 2015.

16. Sonzogni O, Haynes J, Seifried LA, Kamel YM, Huang K, Be Gora MD, Yeung FA, Robert-Tissot C, Heng YJ, Yuan X, et al: Reporters to mark and eliminate basal or luminal epithelial cells in culture and in vivo. PLoS Biol 16: e2004049, 2018.

This work is licensed under a Creative Commons Attribution-NonCommercial-NoDerivatives 4.0 International (CC BY-NC-ND 4.0) License. 